BNL - 66508

\title{
GPLS VME MODULE: A DIAGNOSTIC AND DISPLAY TOOL FOR NSLS MICRO SYSTEMS*
}

\author{
S. Ramamoorthy and J.D. Smith \\ National Synchrotron Light Source \\ Brookhaven National Laboratory \\ Upton, New York 11973-5000
}

April 1999

National Synchrotron Light Source

Brookhaven National Laboratory

Operated by

Brookhaven Science Associates

Upton, NY 11973

Under Contract with the United States Department of Energy

Contract Number DE-AC02-98CH10886 


\section{DISCLAIMER}

This report was prepared as an account of work sponsored by an agency of the United States Government. Neither the United States Government nor any agency thereof, nor any of their employees, nor any of their contractors, subcontractors or their employees, makes any warranty, express or implied, or assumes any legal liability or responsibility for the accuracy, completeness, or any third party's use or the results of such use of any information, apparatus, product, or process disclosed, or represents that its use would not infringe privately owned rights. Reference herein to any specific commercial product, process, or service by trade name, trademark, manufacturer, or otherwise, does not necessary constitute or imply its endorsement, recommendation, or favoring by the United States Government or any agency thereof or its contractors or subcontractors. The views and opinions of authors expresses herein do not necessarily state to reflect those of the United States Government or any agency thereof. 


\title{
GPLS VME MODULE: A DIAGNOSTIC AND DISPLAY TOOL FOR NSLS MICRO SYSTEMS*
}

\author{
S.Ramamoorthy, J.D.Smith, BNL, Upton, NY
}

\begin{abstract}
The General Purpose Light Source VME module is an integral part of every front-end micro in the NSLS control system. The board incorporates features such as a video character generator, clock signals, time-of-day clock, a VME bus interrupter and general-purpose digital inputs and outputs. This module serves as a valuable diagnostic and real-time display tool for the micro development as well as for the final operational systems. This paper describes the functions provided by the board for the NSLS micro control monitor software.
\end{abstract}

\section{INTRODUCTION}

The front-end computers that interface with the hardware in the NSLS control system [1] are VME-based microprocessor systems. The minimum requirements to configure a system are a CPU board with Ethernet controller (68k series: Motorola mvme162 or power PC series: Motorola mv1603, mv1604, mv230x are used at present), one megabyte battery-backed-up memory board and a General Purpose Light Source board (referred to as GPLS board). Additional boards are dictated by the specific hardware or equipment to be controlled and/or monitored. These systems are driven by real-time software known as the NSLS Control Monitor [2],[3]. This paper describes the hardware features of the GPLS board and how the software uses the various components.

\section{HARDWARE DESCRIPTION}

The GPLS board in use since 1987, was originally developed by the NSLS controls and diagnostic groups to provide bus interrupter and ASCII video display functions. As some of the components used by the board have become obsolete, a new GPLS board has been redesigned by Apogee Lab Inc.[4], following the specifications from the NSLS controls group. The new board (Apogee Lab: Model number VME-TVI) provides new features such as a time code reader and generator and enhanced video functions. The board can be accessed through an extended (A32:D16/D08) or a standard (A24:D16/D08) address space of a VME-bus slave interface. The main components of the board are an interrupt generator, a video generator, a timer module and general-purpose inputs and outputs.

*Work performed under the auspices of U.S. Dept. of Energy under the contact no: DE-AC02-98CH10886.

\subsection{Interrupt Generator}

The interrupt generator on the board is capable of generating four interrupts on the VME bus. The input pins for the TTL level signals are on the P2 connector. Each interrupt can be independently enabled or disabled by software. The trigger edge (rising or falling edge of the input signal) and the interrupt level are independently programmable for each interrupt. All the four interrupts can share the same level.

\subsection{Video Generator}

The video section of the board can generate ASCII video that can be displayed on a TV monitor. The board can accept an NTSC signal from an external source and overlay the text data onto the original signal. It uses Dual Ported RAM that permits simultaneous VME-bus access with video character generation. The RAM provides 4 video pages and three memory pages (4 Kbytes each) for general- purpose use. Page selection can be done using the slider switch at the front panel. The video memory can be read and written at any time but only the selected page will be displayed. The video hardware provides four screen formats (12 lines/16 char, 12 lines/20 char, 16 lines/32 char and 16 lines/44 char). It has four programmable color palettes. Each color palette can be set up to one of 64 color shades (e.g. A value 0 will set the color palette to black, 63 to white, 3 to red, 12 to green, 48 to blue and so on). Foreground or background color can be programmed on a character by character basis. The character generator PROM on the board can be programmed for user-definable fonts.

\subsection{Timer Module}

The time module provides three clock signals $(1 \mathrm{kHz}, 4$ $\mathrm{kHz}$ and $250 \mathrm{~Hz}$ ) on the $\mathrm{P} 2$ connector and a time code reader and generator. The time generator can be operated in two modes. In the standalone mode, one can load Time into the registers of the generator and start it by software. In the synchronized mode, an external signal (amplitude modulated $1 \mathrm{kHz}$ IRIG-B carrier) is input through the P2 connector. If the IRIG-B input is lost or corrupted, the board will free wheel and continues to provide the Time. The Time-of-Day information can be read in three words with one microsecond resolution. 


\subsection{Digital I/O}

The other general purpose components are 16-bit LEDs on the front panel, two banks of DIP switches, each consisting of 8 switches and $32 \mathrm{I} / \mathrm{O}$ lines for parallel TTL digital I/O (16 inputs and 16 outputs) on the P2 connector. More information on the board can be obtained from the user's manual supplied by Apogee Labs Inc.[5]. A transition module that will provide easy access to the various signals is also available from the same company.

\section{SOFTWARE INTERFACE}

The control monitor software uses VxWorks which is a commercial real-time operating system. The software consists of a set of system tasks, interrupt handlers, application specific modules and hardware drivers. The system software is standard for all micros and manages the system hardware (GPLS board, etc.), communications, command decoding, etc. It provides system timing, and uses real-time OS primitives to synchronize and coordinate the activities of other tasks. This module isolates all the kernel specifics and system hardware interfacing from application modules and provides services to them for real-time control.

\subsection{Tasks and interrupt handlers}

The system timing has a resolution of 2 milliseconds and it is derived from the $1 \mathrm{kHz}$ clock on the GPLS board. The clock signal is connected to the first interrupt pin to generate the timer interrupt. The monitor provides a wait routine (equivalent to sleep call in Unix) to the application tasks. The tasks can set a wait time as low as 2 milliseconds. The task delay (wait) does not have to rely on the granularity of the time ticks provided by the real-time OS. A second interrupt is generated every 8 milliseconds by connecting the $250 \mathrm{~Hz}$ clock to the second interrupt pin. This interrupt is used both by system and application software to start data acquisition (e.g. reading of $\mathrm{ADC}$ boards) and to signal events to other tasks. The system provides interrupt handlers for 2 external signals (usually from external hardware or from another micro). The external signals are fed to the third and fourth interrupt pins via the transition card. The handlers call the appropriate module to service the interrupt. The levels of these interrupts can be changed by software based on the request from the application tasks.

The use of the GPLS board for generating timing, periodic interrupts and servicing external interrupts has made the software easily portable. Since the software does not use the CPU timers, there is no conflict with the real-time kernel timing. The software has been ported to different 68k series of CPU boards and Power PC boards and also from the RTUX real-time OS to VxWOrks OS with minimum changes in the system module and absolutely no change in the application code.

\subsection{Video Displays}

The video feature of the GPLS board plays a very important role in the monitor software for micro development as well as for the final target systems. The simple MEMORY WRITE operation to generate an ASCII video display is very fast and can be used as a great debugging tool even in interrupt routines. The standard technique of using routines to send messages to a console (e.g. logtask in VxWorks) for diagnostic output is not sometimes suitable in interrupt or bus exception handlers. The system may crash even before the console output is initiated. Also programmers may have encountered cases where a software bug appears to have been removed by introducing the standard printf function for debugging. Since the console output is not synchronous with the code being executed, one can get a false indication of the bug location in the code when it crashes. The simple memory write on the video page exactly locates the problem area in the code.

The software multiplexes the first hardware page to 8 software pages. When hardware page 0 is selected, the software will display one of the eight pages. The software page for the display can be changed easily either by a push-button on a panel connected to the micro or by a remote command from any workstation in the control system network. Both system and application tasks generate displays for diagnostics and for continuous monitoring of device parameters (analog read backs and digital status). Error conditions can be highlighted in yellow and red colors. Figures 1 to 3 show some typical displays from the micros.

Since the board generates composite video (NTSC signal), one can monitor the displays on the local cable TV channels from anywhere in the lab. Some micros whose displays are crucial for operations have dedicated TV channels. Displays from other micros are routed through multiplexers to two general-purpose TV channels. The required display can be selected from any workstation. Since these displays are generated locally, the parameter updates are as fast as they are acquired and there is zero loading on the network.

The hardware page 1 is used by the system task to dump the stack registers and other pertinent information captured when a micro encounters bus errors. Before resetting the micro, one can get some information on the crash from the display. Pages 2 and 3 are used by applications to display the hardware initialization and other diagnostics.

\subsection{Time stamping}

The board extracts the time-of-day information from the amplitude-modulated $1 \mathrm{kHz}$ IRIG-B carrier available from one of the outputs of a Spectracom NetCLOCk/2. 
This derives time from the atomic time standard at the National Institute of Standards and Technology via their WWVB radio broadcast at $60 \mathrm{kHz}$. The system module reads the three timer-registers every 250 milliseconds, decodes the Time-of-Day information and makes it available to other tasks. In addition, the time registers can be used to time a code segment (with an accuracy of + or - 4 microseconds) and display the value on a video page. The time data is also used to time stamp data or an event such as an occurrence of an alarm.

\subsection{Digital I/O interface}

The 16 LEDs are used for diagnostic purposes. Each task sets up different LEDs. This provides a quick indication if a task gets into a loop. One LED is used to generate heartbeat signal by application tasks.

The DIP switches are used for setting up different configurations or modes of operation. One bank of switches is located on the board and is used to define configurations that cannot be accidentally altered. The front panel switches can be changed easily for the required mode of operation. As an example, one switch is assigned for controlling the parameter initialization during start-up. The micros save the parameters last set by the operators from a workstation, in the batterybacked-up memory. When the micro reboots following a "reset", "power-on" or "power-dip", it retrieves the battery-backed-up data for or the default values based on the switch setting. Another switch is used to inhibit the reporting of alarm messages.

The digital inputs and outputs are used by the applications for various purposes. One of the outputs is connected to the system $\boldsymbol{R} \boldsymbol{E S E T}$ pin. Using a software command one can initiate a system RESET. The outputs have also been used to generate interrupts by software to other micros or external hardware.

\section{CONCLUSIONS}

The use of the GPLS board timers for system timing and interrupts by the software makes the porting of software to different VME-based CPU boards and to different realtime operating systems very easy. The video display serves as a great debugging tool during program development. The fast display of signal values and hardware status on TV without any load on the network are widely used for diagnostics and general monitoring by engineers, operators and physicists.

\section{ACKNOWLEDGEMENT}

The authors wish to thank Gary Frisbie for his technical support during the testing of the boards and to Dave Grebe of Apogee Lab for his constructive suggestions.
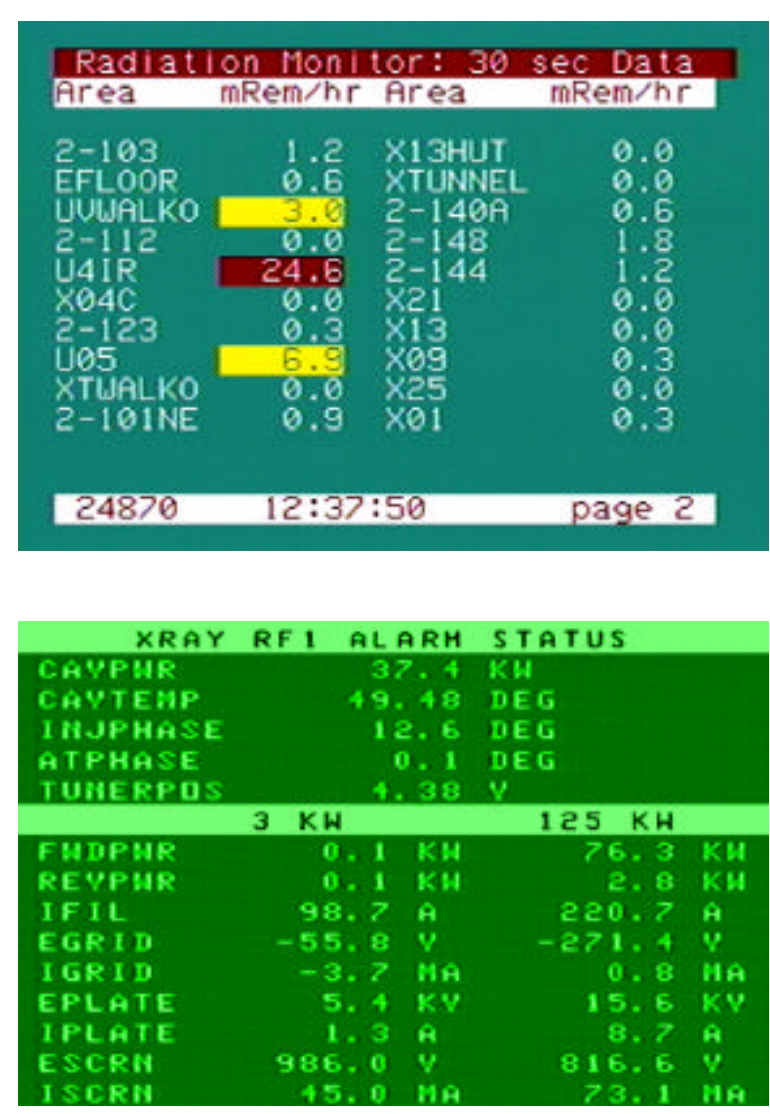

Figure 1. Display of radiation levels.

Figure 2. Display of XRF1 signals.

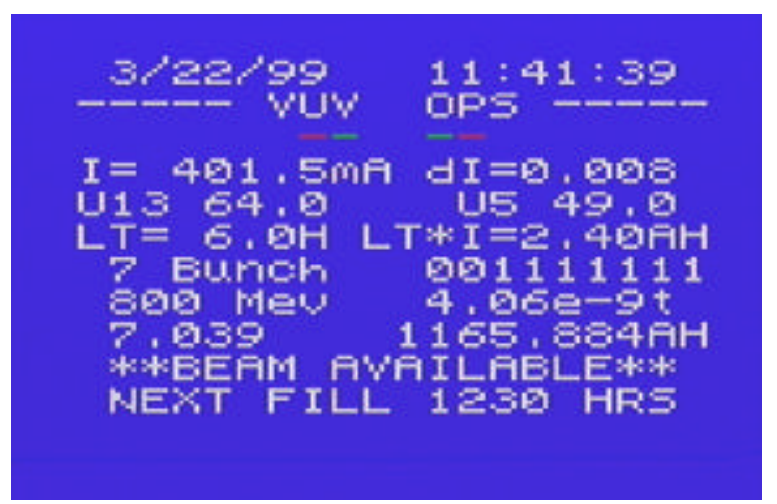

Figure 3. Display of VUV ring Parameters.

\section{REFERENCES}

[1] J.D.Smith, S.Ramamoorthy, Y.Tang, Nucl.Inst. and Meth.In Phys. Res, A32 (1994) 114.

[2] S.Ramamoorthy, J.D.Smith, Proc.IEEE PAC 1993, 1849.

[3] http://www.nsls.bnl.gov/Systems/Controls

[4] Apogee Lab Inc.,Dickerson Rd, Unit3/4, North Wales, PA19454.

[5] User's Manual for Time/Video/Interrupt Module Model VME-TVI. 